\title{
Emerging trends of applied neural computation
}

\author{
Lazaros lliadis $^{1} \cdot$ Ilias Maglogiannis ${ }^{2}$
}

Published online: 21 March 2020

(C) Springer-Verlag London Ltd., part of Springer Nature 2020

The developments and the upward trend of neural computation (NCO) in the first two decades of the twenty-first century have introduced important changes in our postmodern societies. They have brought reorientation and reconceptualization of the way that we are interacting, communicating, thinking and working. Dramatic revolutions have been shaped in many diverse scientific areas.

This is the editorial of the "Emerging Trends of Applied Neural computation" Special Issue of the NCA Journal. It presents timely evolutions and developments of $\mathrm{NCO}$ algorithms, and it demonstrates their wide spectrum of applications. It includes eleven high-quality scientific research papers, presenting innovative research, falling in this area. They have been selected after passing successfully through a peer review process by independent academic referees.

The first paper "An Adaptive Ensemble Classification Framework for Real-Time Data Streams by Distributed Control Systems" is authored by Wang Sufang, from the Department of Information Engineering, Jiaozuo College, China.

This scientific research proposes a sophisticated adaptive ensemble classification framework for real-time data streams. It employs "kappa architecture," and it is based solely on advanced computational intelligence methods. It concerns the digital security of smart grids which are critical infrastructure networks, playing a decisive role for the survival of economy. The range of threats is enhanced

Lazaros Iliadis

liliadis@civil.duth.gr

Ilias Maglogiannis

imaglo@unipi.gr

1 Laboratory of Mathematics and Informatics (ISCE), Department of Civil Engineering, School of Engineering, Democritus University of Thrace, University Campus, Kimmeria, 68200 Xanthi, Greece

2 Department of Digital Systems, University of Piraeus, 80, M. Karaoli \& A. Dimitriou Street, 18534 Piraeus, Greece by the heterogeneity of smart grids which are incorporated in industrial control systems (e.g., SCADA, distributed control systems and programmable logic controllers). The proposed approach has proven to have many advantages and to perform very efficiently.

The second paper is authored by Georgios Drakopoulos and Phivos Mylonas, from the Department of Informatics, of the Ionian University, Corfu, Greece. The title is "Evaluating Graph Resilience with Tensor Stack Networks: A Keras Implementation."

In communication networks, structural coherency, namely the ability to maintain total connectivity even after some data links are lost for an indefinite time, is a major design consideration. This research treats communication systems in an abstract level as graphs, where the existence of an edge depends heavily on the local connectivity properties between two nodes. The authors are employing tensor stack networks (TSNs) to evaluate the resilience of graphs by considering their actual structural strength. TSNs are an emerging deep learning classification methodology for big data which can be expressed either as stacked vectors or matrices. The Estrada and the odd Estrada indices and also the clustering coefficient metrics are employed for validation. The obtained TSN classification model is evaluated by six metrics, and its efficiency is proved.

Jie Jin, Lv Zhao, Mu Li, Zaifang Xi, from the School of Information and Electrical Engineering, Hunan University of Science and Technology, Xiangtan, China, and Fei Yu from the College of Computer and Communication Engineering, Changsha University of Science and Technology, Changsha, China, are the authors of the third paper. It is entitled "Improved zeroing neural networks for finite time solving nonlinear equations."

Nonlinear equations are an important cornerstone of nonlinear science, and many practical problems in scientific and engineering fields can be described by them. This paper introduces an improved zeroing neural network (IZNN) model that can solve the time-invariant nonlinear equation (TINE) and the time-varying nonlinear equation 
(TVNE) in predictable and finite time. The IZNN has proven to be more efficient, stable and reliable for solving TVNE than the existing ZNN approach. Moreover, its convergence time is finite, and it can be estimated. The authors clearly present the theoretical and numerical simulation results, and the superiority and effectiveness of the IZNN model is shown.

An interesting research entitled "An improved weightconstrained neural network training algorithm" is authored by Ioannis E. Livieris and Panagiotis Pintelas, from the University of Patras, Greece.

The authors of this interesting paper are introducing an improved weight-constrained neural network training approach, under the name iWCNN. The proposed algorithm exploits the numerical efficiency of the "limitedmemory" Broyden, Fletcher, Goldfarb, Shanno matrices (L-BFGS), by applying a gradient-projection strategy for handling the bounds on the weights. Moreover, an attractive property of the iWCNN is that it utilizes a new scaling factor for defining the initial Hessian approximation (HEA) used in the L-BFGS formula. Specifically, HEA is defined utilizing a small number of correction vector pairs. The final aim is to further exploit them in order to increase the efficiency of the training algorithm and the convergence rate of the minimization process. The preliminary numerical experiments provide empirical evidence that the proposed training algorithm accelerates the training process.

The fifth paper is "Genetic and Deep Learning clusters based on Neural Networks for management decision structures" by Will Serrano, Imperial College of London, UK.

Human and business management decisions are made following a structured approach. A hierarchical process is involved that requires a level of compromise between risk, cost, reward, experience and knowledge. This research paper introduces a management decision structure, emulating the function of the human brain. It is based on genetic and deep learning (DEL) clustering algorithms and on random neural networks (RNN). Reinforcement learning takes quick and specific local decisions, DEL clusters provide identity and memory, and DEL management clusters make final strategic decisions. The employed genetic algorithm (GNA) transmits the learned information to future generations in the network weights. The subject's information is a combination of memory, identity and decision data, so it is never lost but transmitted. Thus, the GNA provides immortality. The management decision structure has been applied and validated in a smart investment "Fintech" application with rewarding results.

Petia Koprinkova-Hristova from the Institute of Information and Communication Technologies, Bulgarian Academy of Sciences, Sofia, Bulgaria, Miroslava Stefanova, Bilyana Genova, and Nadejda Bocheva, from the
Institute of Neurobiology, Bulgarian Academy of Sciences, Sofia, Bulgaria, and Radoslava Kraleva, Velin Kralevare from the South West University, Blagoevgrad, Bulgaria, are the authors of the sixth paper. The title is "Features Extraction from Human Eye Movements via Echo State Network."

The authors of this research paper are introducing a novel feature extraction approach, from eye movement's time series, aimed at age-related classification of humans. Their model exploits the properties of the echo state network (ESN) reservoir state, which is achieved after tuning its intrinsic plasticity. Eye tracker recordings of human eye movements were fed into the ESN in order to be used in visual stimulation and decision making processes. The results support the view that the metrics and dynamics of the eye movements depend on age to a certain degree, though they are more strongly related to the visual stimulation characteristics.

An interesting research entitled "Continuous Drone Control using Deep Reinforcement Learning for Frontal View Person Shooting" is described in the seventh paper. The authors are Nikolaos Passalis and Anastasios Tefas from the Department of Informatics of the Aristotle University of Thessaloniki, Greece.

Using drones for aerial cinematography requires the coordination of several people, which increases the cost and reduces shooting flexibility. Moreover, this raises the cognitive load of drone operators. The authors of this research paper aim to overcome these limitations. More specifically, they propose a deep reinforcement learning (RL) method for continuous fine-grained drone control that allows acquiring of high-quality frontal view person shots. A head pose image dataset is combined with 3D models and face alignment/warping techniques to develop an RL environment that realistically simulates the effects of the drone control commands. A reward-shaping approach is also proposed to improve the stability of the employed model. Apart from performing continuous control, it is demonstrated that the introduced method can be also effectively combined with simulation environments that support only discrete control commands, improving control accuracy. The effectiveness of the proposed technique is experimentally demonstrated using several quantitative and qualitative experiments.

The eighth paper is entitled "Spam Detection on Social Networks using Cost-Sensitive Feature Selection and Ensemble-Based Regularized Deep Neural Networks," and it is authored by Aliaksandr Barushka and Petr Hajek from the University of Pardubice, Czech Republic.

The global wide spread of social networks has introduced the need for the development of sophisticated filters to deal with spam detection. This is a complex problem of increasingly importance. The authors of this paper are 
introducing a novel cost-sensitive approach to social network spam filtering that outperforms traditional classifiers. The introduced approach comprises of two stages: In the first stage, multi-objective evolutionary feature selection is employed to minimize both the misclassification cost and the number of attributes necessary for spam filtering. Then, cost-sensitive ensemble learning techniques are applied, with regularized deep neural networks as base learners.

Nikolaos Kolokas, Anastasios Drosou and Dimitrios Tzovaras from the Center for Research and Technology Hellas Thessaloniki, Greece, are the authors of the ninth paper "Text Synthesis from Keywords: A Comparison of Recurrent-Neural-Network-based Architectures and Hybrid Approaches."

This paper presents a novel approach for text synthesis in word level, with the use of keywords. A speech tagging library is employed to extract verbs and nouns from the considered texts. A recurrent neural network is developed and employed to map the keyword sequence to the entire text. Successive reformulations of the keyword and full text word sequences are performed, in order to serve as input and output of the network. The predicted texts are meaningful enough, and the performance depends on the difficulty of the case, which is determined by the percentage of full text words considered as keywords, which ranges from 33 to $50 \%$ approximately. Another important factor is training memory cost, which is mainly affected by the architecture of the network and by the similarity between different texts.

The tenth paper "Deep Bayesian Self-Training" is authored by Fabio De Sousa Ribeiro, Francesco Calivá, Georgios Leontidis and Stefanos Kollias from the MLearn Group School of Computer Science, University of Lincoln, UK, and Mark Swainson, Kjartan Gudmundsson from the National Center for Food Manufacturing, Holbeach Technology Park, UK.

Supervised deep learning has been highly successful in recent years, achieving state-of-the-art results in most tasks. However, with the ongoing uptake of such methods in industrial applications, the requirement for large amounts of annotated data is often a challenge. In most real-world problems, manual annotation is practically intractable due to time/labor constraints; thus, the development of automated and adaptive data annotation systems is highly sought after. This paper proposes a deep Bayesian self-training methodology for automatic data annotation, by leveraging predictive uncertainty estimates. The introduced approach is employing neural network (NN) architectures of varying inference and a practical adaptation procedure for handling high label variability between different dataset distributions. This is achieved through clustering of NN latent variable representations; experimental application on both public and private datasets has proved the efficiency of the developed approach and its superiority over standard self-training baselines.

Finally, the last paper is entitled "Spatiotemporal Neural Networks for Action Recognition Based on Joint Loss," and it is authored by Chao Jing, Ping Wei, Hongbin Sun and Nanning Zheng, from the Xi'an Jiaotong University, China.

Action recognition is a challenging and important task in a myriad of significant fields, such as intelligent robots and video surveillance. Deep learning and a variety of neural network techniques have been widely applied to action recognition and attained remarkable results. However, it is still difficult to recognize actions in complicated scenes, containing motions of high similarity, various illumination conditions and background noise. The authors of this paper present a spatiotemporal neural network approach with "joint loss," to recognize human actions from videos in spatial dimension. The introduced model comprises of a "two stream-based" network, extracting optical flow and appearance features from each video frame. Moreover, it employs a group of "long short-term memory" structures following the spatial network, which describes the required temporal and transition information. The spatiotemporal neural network model has been trained using video samples from two challenging datasets, and it has proven its high level of efficiency.

We wish to express our appreciation and deep gratitude to the Editor in Chief of the Springer Neural Computing and Applications Journal, Professor John Macintyre, for his full support and for offering us the privilege to edit a special issue in this high-quality journal. We have tried to make a minor and timely contribution to the existing literature. We hope that the novel approaches presented in this publication will be appreciated by the international scientific community. We are certain that they will inspire further research on neural computing and applications in several and diverse domains.

The Guest Editors Professor Lazaros S. Iliadis Professor Ilias Maglogiannis

Publisher's Note Springer Nature remains neutral with regard to jurisdictional claims in published maps and institutional affiliations. 